\title{
Aortic propagation velocity in the prediction of coronary artery disease severity
}

\author{
Eftal Murat Bakircia , Husnu Degirmencia, Hakan Duman ${ }^{b}$, Selami Demirellic, Hikmet Hamura, Mutlu Buyuklua, \\ Gokhan Ceyhun ${ }^{d}$, Ergun Kutlusoy ${ }^{\mathrm{a}}$
}

\begin{abstract}
Aims. To investigate the association of the aortic propagation velocity (APV) with coronary artery disease (CAD) in patients with stable angina pectoris (SAP) through SYNTAX scores (SS).

Methods. The study population comprised 214 SAP subjects who received a coronary angiography. The APV and carotid intima-media thickness (CIMT) were examined and SS was calculated. Subjects were grouped following specific SS criteria: SS less than 22 (low) and SS greater than or equal to 22 (high).

Results. High SS subjects had lower APV compared to low SS [39.0 (32.0-51.7) vs. 55.0 (45.0-62.0) cm/s, respectively; $P<0.001]$ and higher CIMT ( $0.86 \pm 0.24$ vs. $0.74 \pm 0.21 \mathrm{~mm}$, respectively; $P<0.001)$. APV demonstrated a negative correlation with the CIMT $(r=-0.239, P<0.001)$, age $(r=-0.188, P=0.006)$, and $\mathrm{SS}(r=-0.561, P<0.001)$ and showed a positive association with LV ejection fraction ( $r=0.163, P=0.017)$. APV, CIMT, diabetes, low-density lipoprotein cholesterol (LDL-C), and age were determined to be markers independently of a high SS.

Conclusion. APV, CIMT, diabetes, LDL-C and age are independently linked to the CAD severity of SAP subjects. Decreased APV, an indicator of subclinical atherosclerosis, may independently help determine the severity of atherosclerotic CAD in SAP patients.
\end{abstract}

\section{Key words: APV, CIMT, CAD, SYNTAX score}

Received: October 3, 2020; Revised: December 5, 2020; Accepted: January 7, 2021; Available online: February 22,2021 https://doi.org/10.5507/bp.2021.001

(c) 2022 The Authors; https://creativecommons.org/licenses/by/4.0/

\author{
${ }^{a}$ Department of Cardiology, Faculty of Medicine, Erzincan Binali Yildirim University, Erzincan, Turkey \\ ${ }^{b}$ Department of Cardiology, Faculty of Medicine, Recep Tayyip Erdogan University, Rize, Turkey \\ 'Department of Cardiology, Kayseri Education and Research Hospital, Kayseri, Turkey \\ ${ }^{d}$ Department of Cardiology, Faculty of Medicine, Ataturk University, Erzurum, Turkey \\ Corresponding author: Eftal Murat Bakirci, e-mail: eftalka@yahoo.com
}

\section{INTRODUCTION}

Atherosclerosis, a multifactorial disease, can complicate any part of the arterial system ${ }^{1}$. Prevention is one of the main principles of healthcare. Non-invasive imaging techniques have been used to identify atherosclerosis and improve clinical outcomes. It has been shown that augmented CIMT is atherosclerosis and cardiovascular risk predictor ${ }^{2}$. Furthermore, propagation velocity of the descending thoracic aorta (APV) has been linked to coronary/carotid atherosclerosis ${ }^{3}$.

The SYNTAX score (SS) has been used to score the CAD severity. It scores atherosclerotic lesion characteristics as well as the coronary artery anatomy. Substantial clinical findings have determined that the SS is reliable for selecting optimal revascularization in CAD patients ${ }^{4}$. Several studies have shown that the higher SS is directly proportional the rate of acute and long-term cardiovascular events ${ }^{5-7}$. Using noninvasive imaging, patients that are high risk for cardiovascular events/CAD severity can be identified early. An increase in CIMT has been linked to complexity and severity of CAD $\left(\right.$ ref. $\left.^{8}\right)$. To our knowledge, no reports have been published investigating the association between the APV and CAD complexity. Therefore, we aimed to investigate the association of APV with $\mathrm{CAD}$ severity in stable angina pectoris (SAP) patients using the SS system.

\section{MATERIALS AND METHODS}

A total of 214 SAP subjects that had a coronary angiography (CAG) were recruited for the study. The exclusion criteria were acute coronary syndrome; an SS of 0 ; a history of myocardial infarction, coronary artery bypass grafting or percutaneous coronary intervention; renal insufficiency; hepatobiliary complications; alcohol consumption; acute/chronic inflammation or infection; aortic aneurysm; severe valvular disease; left ventricular systolic dysfunction; atrial fibrillation; and use of steroids. Approval of the study granted by the local ethics committee, and subjects provided consent before participation.

Following a full workup, patients were asked to undergo an echocardiographic exam. Blood samples (at fasting) for biochemical, lipid, and hematological analysis were collected 1 day before CAG. The eGFR was determined using the Cockcroft-Gault formula.

\section{Echocardiographic analysis}

Echocardiography was done via GE Vivid S5 system and a $2.5-\mathrm{MHz}$ phased-array transducer. Images, following a complete transthoracic echocardiographic exam, were taken from the left lateral decubitus position. All exams were done by a blinded, experienced cardiologist. The LVEF was determined by modification of the Simpson method. Following the echocardiography, color M-mode 

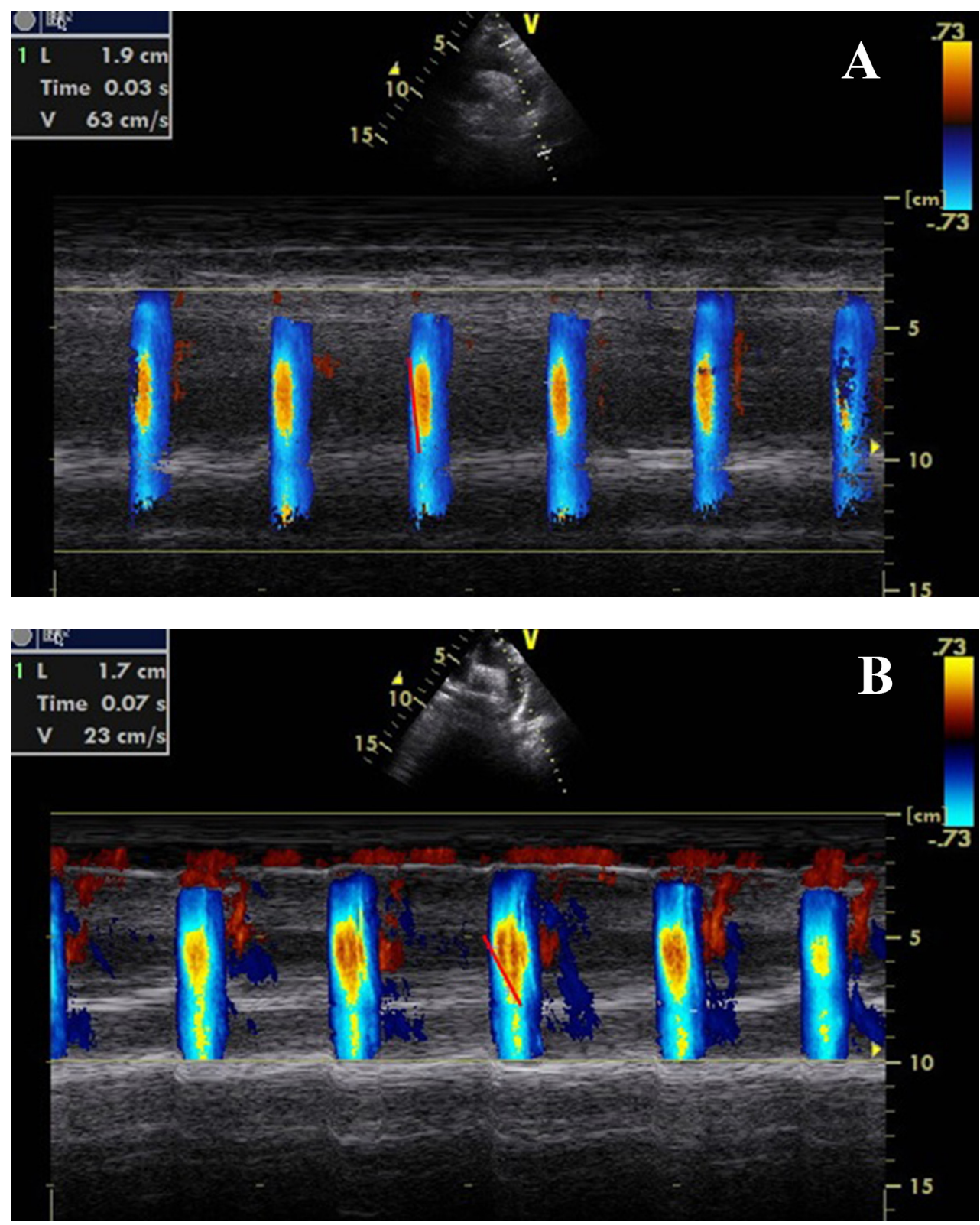

Fig. 1. Descending aorta propagation velocity in a low SS (A) and high SS (B) subject.

Doppler was recorded from a suprasternal window with parallel to the descending aorta main flow. An adaptation of the Nyquist limit (30 to $50 \mathrm{~cm} / \mathrm{s}$ ) was performed. M-mode spatiotemporal velocity map was obtained (Fig. $1 \mathrm{~A}$ and $\mathrm{B})$. Next, aliasing was performed to adjust the velocity and a clear velocity slope was achieved. The APV was determined by dividing the distance by propagation slope time of the velocity slope.

\section{Assessment of CIMT}

CIMT was determined by a Toshiba Diagnostic Ultrasound System machine and a 7.5-MHz transducer, while subjects were in the supine position. The technique was accordance with the American Society of Echocardiography CIMT Task Force ${ }^{9}$. Briefly, CIMT was demarcated from the edge of the lumen-intima to the media-adventitia on the outer wall of the common carotid artery. The carotid artery transverse and longi- tudinal planes were subjected to CIMT measurement $\sim 1$ centimeter proximal to the carotid artery bifurcation in the free plaque area.

\section{Angiographic analysis}

Selective CAG was done using the Judkins technique. Individual angiograms were evaluated by 2 independent and qualified blinded cardiologists. A coronary lesion of greater than or equal to $50 \%$ diameter stenosis in 1.5 $\mathrm{mm}$ vessels was used for scoring. Verson (2.11) of the SS software used for classification [SS $<22$ (low) and $\mathrm{SS} \geq 22$ (high)].

\section{Statistical Analysis}

Data were analyzed using SPSS v17 (SPSS Inc., Chicago, IL, USA). The One-Sample KolmogorovSmirnov test was used to determine continuous variable distribution normality. Between groups statistical differ- 
Table 1. Clinical and Demographic information of subjects with low and high SYNTAX Scores

\begin{tabular}{|c|c|c|c|}
\hline & $\begin{array}{l}\text { Low Syntax Score } \\
(<22),(n=134)\end{array}$ & $\begin{array}{l}\text { High Syntax Score } \\
(\geq 22),(n=80)\end{array}$ & $P$ \\
\hline Age, years, $\mathrm{n}(\%)$ & $51.5(48-64)$ & $62(54-67)$ & $<0.001$ \\
\hline Sex, male, $n(\%)$ & $103(76.9 \%)$ & $70(87.5 \%)$ & 0.056 \\
\hline Diabetes, $n(\%)$ & $27(20.1 \%)$ & $32(40 \%)$ & 0.002 \\
\hline Hypertension, n(\%) & $53(39.6 \%)$ & $33(41.3 \%)$ & 0.806 \\
\hline Smoking, $\mathrm{n}(\%)$ & $65(48.5 \%)$ & $35(43.8 \%)$ & 0.500 \\
\hline BMI, $\mathrm{kg} / \mathrm{m}^{2}$ & $28.5 \pm 4.9$ & $29.5 \pm 5.8$ & 0.186 \\
\hline LVEF on admission, $\%$ & $54(48-62)$ & $54(47.2-56)$ & 0.152 \\
\hline Hemoglobin, g/dL & $13.6 \pm 2.4$ & $13.9 \pm 2.1$ & 0.435 \\
\hline White blood cell count, $\times 10^{9} / \mathrm{L}$ & $6.7(6.1-8.2)$ & $6.8(6.1-8.3)$ & 0.978 \\
\hline Platelet count, $\times 10^{9} / \mathrm{L}$ & $278(214-353)$ & $290(198-354)$ & 0.819 \\
\hline $\mathrm{eGFR}<60, \mathrm{ml} / \mathrm{min} / 1.73 \mathrm{~m}^{2}, \mathrm{n}(\%)$ & $7(5.2 \%)$ & $11(13.8 \%)$ & 0.030 \\
\hline LDL cholesterol, mg/dL & $126.7 \pm 30.1$ & $131.9 \pm 31.1$ & 0.232 \\
\hline HDL cholesterol, mg/dL & $38.2 \pm 7.8$ & $36.3 \pm 7.3$ & 0.070 \\
\hline Triglyceride, mg/dL & 143(83-199.2) & $134(80-186.7)$ & 0.449 \\
\hline \multicolumn{4}{|l|}{ Previous medications, $\mathrm{n}(\%)$} \\
\hline Aspirin & $24(17.9 \%)$ & $15(18.8 \%)$ & 0.878 \\
\hline ACEi/ARB & $33(24.6 \%)$ & $19(23.8 \%)$ & 0.885 \\
\hline Beta-blocker & $17(12.7 \%)$ & $9(11.3 \%)$ & 0.756 \\
\hline Statin & $22(16.4 \%)$ & $11(13.8 \%)$ & 0.601 \\
\hline CIMT, mm & $0.74 \pm 0.21$ & $0.86 \pm 0.24$ & $<0.001$ \\
\hline $\mathrm{APV}, \mathrm{cm} / \mathrm{sec}$ & $55(45-62)$ & $39(32-51.7)$ & $<0.001$ \\
\hline
\end{tabular}

ACE-i/ARB, angiotensin converting enzyme inhibitor/angiotensin receptor blocker; APV, aortic propagation velocity; BMI, body mass index; CIMT, carotid intima-media thickness; eGFR, estimated glomerular filtration rate; HDL, high-density lipoprotein; LDL, low-density lipoprotein; LVEF, left ventricular ejection fraction.

ences were determined by Student $t$ test or Mann-Whitney $\mathrm{U}$ test. Variables that were continuous were given as mean \pm SD or median (interquartile range). Categorical data were subjected to Pearson's chi-square or Fisher exact test. Data considered categorical were presented as number of cases (percentage). Pearson product-moment or Spearman's rank correlation coefficients were used to determine the association of continuous variables. Multiple logistic regression analysis with the backward LR method was used to determine the independent predictors of a high SS $(P<0.25)$. A p-value of less than 0.05 was considered statistically significant.

\section{RESULTS}

A total of 214 SAP subjects (mean age $59.3 \pm 12.6$ years; $80.8 \%$ male) were recruited for the study. One hundred thirty four subjects were categorized into the low SS group and 80 subjects in the high SS group. Clinical, laboratory, and echocardiographic findings from both groups are presented in Table 1. Subjects with high SS were significantly older and more commonly had DM. Subjects with low eGFR was higher in the high SS group. Subjects with low SS had a significantly higher median APV compared to the high SS subjects [55.0 (45.0-62.0) $\mathrm{cm} / \mathrm{s}$ vs 39.0 (32.0-51.7); $P<0.001$ ] (Fig. 2). The high SS group had augmented CIMT measurements $(0.86 \pm 0.24$ vs. $0.74 \pm 0.21 \mathrm{~mm}$, respectively; $P<0.001)$ compared to the low SS.

APV was negatively associated with the CIMT $(\mathrm{r}=-0.239, P<0.001)$, age $(\mathrm{r}=-0.188, P=0.006)$, and SS $(\mathrm{r}=-0.561, \mathrm{p}<0.001)$ but positive with the LVEF $(\mathrm{r}=0.163$, $P=0.017)$.

Accordingly, the APV (OR, 0.913; 95\% CI, 0.8860.941; $P<0.001$ ), CIMT (OR, 1.017; 95\% CI, 1.002-1.033; $P=0.029)$, age (OR, 1.056; 95\% CI, 1.027-1.087; $P<0.001)$, DM (OR, 3.034; 95\% CI, 1.374-6.702; $P=0.006$ ), and LDL cholesterol (OR, 1.014; 95\% CI, 1.002-1.026; $P=0.027)$ were identified as independent identifiers of high SS (Table 2).

\section{DISCUSSION}

In this report, APV, CIMT, advanced age, LDL cholesterol level, and presence of DM were shown to independently determine CAD severity as assesed by SS in patients with suspected CAD. Furthermore, we found a significant correlation between the CIMT, a marker of subclinical atherosclerosis, and the APV. To our knowl- 
Table 2. Predictors of high SS.

\begin{tabular}{lllr}
\hline Variables & Wald & OR $(95 \%$ CI $)$ & $P$ \\
\hline Step 1 (baseline model) & & & \\
Age & 12.873 & $1.057(1.025-1.089)$ & $<0.001$ \\
Gender & 0.607 & $1.483(0.551-3.993)$ & 0.436 \\
Presence of DM & 5.390 & $2.638(1.163-5.984)$ & 0.020 \\
BMI & 0.389 & $1.022(0.954-1.096)$ & 0.533 \\
LVEF & 0.637 & $0.980(0.933-1.030)$ & 0.425 \\
eGFR<60 & 0.736 & $1.797(0.471-6.861)$ & 0.391 \\
LDL cholesterol & 5.890 & $1.015(1.003-1.028)$ & 0.015 \\
HDL cholesterol & 0.888 & $0.977(0.929-1.026)$ & 0.346 \\
CIMT & 4.173 & $1.016(1.001-1.032)$ & 0.041 \\
APV & 31.659 & $0.915(0.887-0.944)$ & $<0.001$ \\
Step 6 (final step) & & & \\
Age & 14.082 & $1.056(1.027-1.087)$ & $<0.001$ \\
LDL cholesterol & 4.905 & $1.014(1.002-1.026)$ & 0.027 \\
Presence of DM & 7.536 & $3.034(1.374-6.702)$ & 0.006 \\
CIMT & 4.767 & $1.017(1.002-1.033)$ & 0.029 \\
APV & 34.065 & $0.913(0.886-0.941)$ & $<0.001$ \\
\hline
\end{tabular}

APV, aortic propagation velocity; BMI, body mass index; CIMT, carotid-intima media thickness; DM, diabetes mellitus; eGFR, estimated glomerular filtration rate; HDL, high-density lipoprotein; LDL, low-density lipoprotein; LVEF, left ventricular ejection fraction.

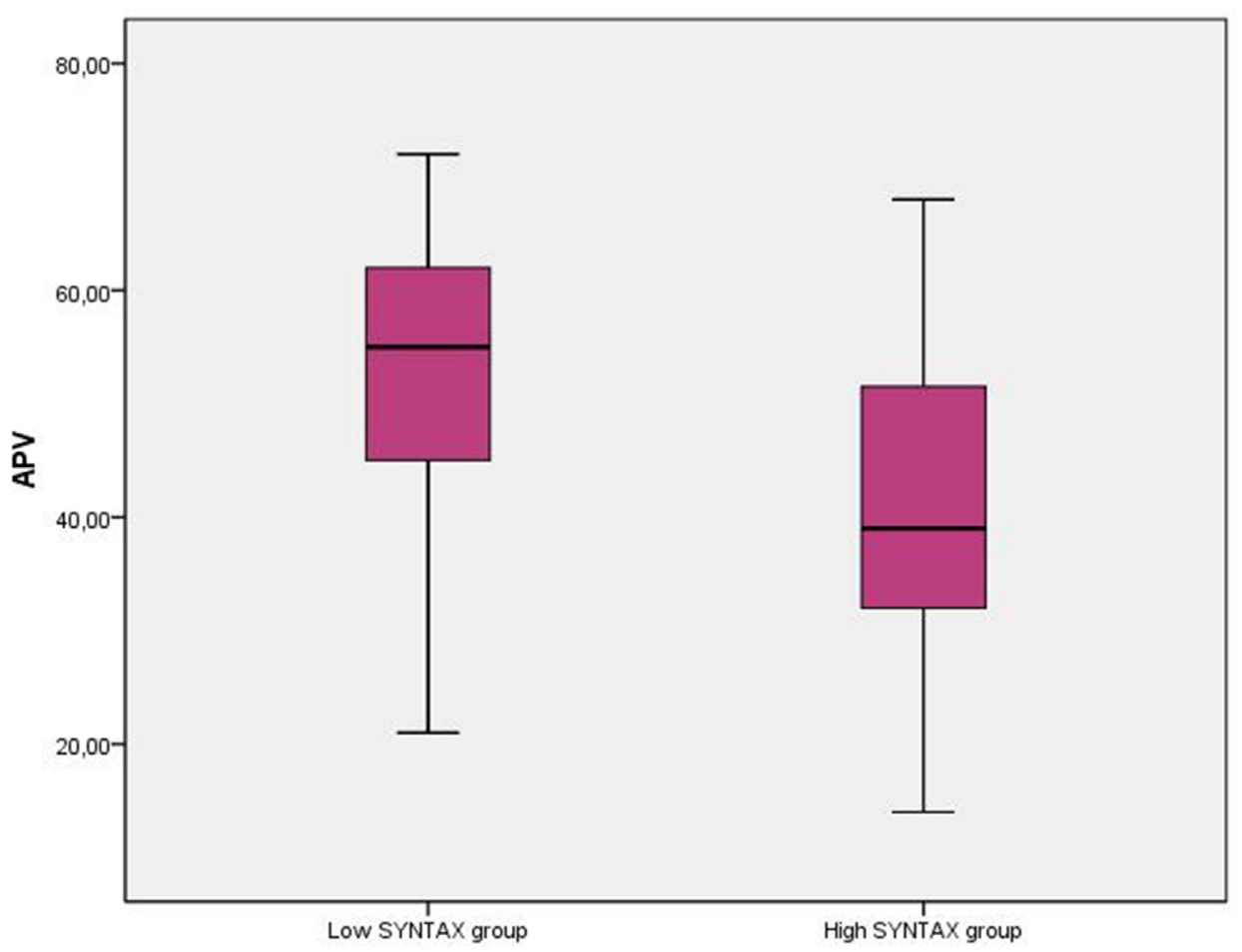

Fig. 2. APV values from low and high SS groups. APV, aortic propagation velocity. 
edge, we are the first to report the association with APV and CAD severity in patients with SAP.

The SS scores atherosclerotic lesion characteristics and coronary artery anatomy and reflects complexity grade and severity of atherosclerosis ${ }^{10}$. SS has been documented to independently predict mortality/major adverse acute and long-term cardiovascular events ${ }^{11,12}$. Thus, prediction of a high SS in patients with SAP before CAG is important in terms of determining appropriate treatment options and for prediction of adverse cardiovascular complications ${ }^{13,14}$. Specific complications, such as diffuse coronary atherosclerosis and severe vascular calcification, have been more commonly observed in the elderly. Additionally, patients with DM have an increased atherosclerotic burden, increased multi-vessel disease rate, and higher numbers of severe lesions ${ }^{15-18}$. The higher SS observed in elderly and diabetic patients can be explained by these pathological changes. As a new result, we observed that the APV is an independent risk factor for a high SS. Mounting reports have suggested that aortic atheroma may help identify generalized atherosclerosis ${ }^{19,20}$. Fazio et al. ${ }^{21}$ showed that atherosclerotic plaques in the thoracic aorta were indicators of CAD. Another report demonstrated that aortic plaques imaged by trans-oesophageal echocardiography had a $93 \%$ sensitivity and $82 \%$ specificity for significant CAD (ref. ${ }^{22}$ ). Atherosclerosis has been shown to escalate arterial resistance by a thickened and stiffened arterial wall. The APV decreases due to the increased resistance that develops secondary to atherosclerosis in the descending thoracic aorta. Thus, the APV decreases as the severity of the atherosclerosis in the descendent aortic artery increases. Clinical use of trans-oesophageal echocardiography for risk determination in patients with CAD in daily practice is not feasible. However, the APV is an easy and simple echocardiographic parameter that can be used to assess the involvement of atherosclerosis in the thoracic aorta. Simsek et al. ${ }^{20}$ reported that DM may be linked to subclinical atherosclerosis as determined by the APV and CIMT and that the APV is correlated significantly and inversely with CIMT. In another study, the APV had the highest ability to predict CAD among the clinical and echocardiographic variables. An APV of less than or equal to $41 \mathrm{~cm} / \mathrm{s}$ had a $82.4 \%$ sensitivity and 97.2\% specificity for predicting CAD (ref. ${ }^{23}$ ). Similarly, Sen et al. ${ }^{24}$ indicated that APV was reduced in 51 CAD subjects in comparison to 42 patients without CAD. The APV was correlated with aortic stiffness parameters and the CIMT. They also used the Gensini score to assess CAD complexity/severity and did not identify any relationship among the APV, Gensini score, and/or number of affected coronary arteries. It can be asserted that the lack of statistically significant correlation in the aforementioned report was related to with the small patient cohort. SS provides better information about the complexity of CAD than does the Gensini score ${ }^{10}$. Similarly, Ghaderi et al. ${ }^{25}$ showed that APV is decreased in $38 \mathrm{CAD}$ patients and is associated with the presence and severity of CAD. In the present study, we used the SS to assess CAD severity/complexity. Although the ranges of APV values were similar between the high and low SS groups, patients with low SS had a significantly higher median APV compared to the high SS patients. Furthermore, we found that APV and SS were independently associated in our larger patient population.

An increased CIMT is a measure of subclinical atherosclerosis and has been linked to enhanced development of CAD in asymptomatic patients ${ }^{26}$. Korkmaz et al. ${ }^{8}$ demonstrated a significant association with the CIMT and SS. Similarly, our study determined that CIMT was an independent predictor of a high SS. Additionally, we observed that APV had a negative correlation with CIMT, a known indicator of atherosclerosis.

It is well known that increased CAD severity is linked to unfavorable acute/long-term cardiovascular complications ${ }^{11,12}$. Using noninvasive techniques to predict CAD severity, at risk patients for increased cardiovascular complications can be identified early. APV and CIMT, which are non-invasive, safe, and inexpensive, may be useful for predicting severity of CAD. Additionally, APV and CIMT may be useful for non-invasive cardiovascular patient risk assessment, management, and prevention.

\section{Study Limitations}

Our study has several limitations. First, our sample size was small. Secondly, the suprasternal image quality of some patients did not allow for clear measurement of the APV. Third, our study was only comprised of SAP patients and excluded subjects with acute coronary syndrome. Finally, our analyses did not involve long-term events.

\section{CONCLUSIONS}

The present study results indicate that a lower of APV, increased CIMT, advanced age, LDL-C, and presence of $\mathrm{DM}$ are independent predictors of CAD severity in subjects with stable CAD. We found that the APV is significantly correlated with CIMT. We suggest that a reduction in APV, an indicator of subclinical atherosclerosis, may independently determine the severity of atherosclerotic CAD in SAP patients. More large-scale prospective study is needed to delineate APV as a screening marker for subjects with severe atherosclerotic CAD.

Author contributions: EMB, HD, HD: study design/drafting the manuscript; $\mathrm{HH}, \mathrm{SD}, \mathrm{MB}$ : statistical analysis; EMB, SD, GC: literature search: EMB, HD, HH, GC, MB: data collection and management; EMB, HD, ET, $\mathrm{MB}, \mathrm{HH}$ : revision of the manuscript.

Conflict of interest statement: The authors state that there are no conflicts of interest regarding the publication of this article. 


\section{REFERENCES}

1. Mallika V, Goswami B, Rajappa M. Atherosclerosis pathophysiology and the role of novel risk factors: a clinicobiochemical perspective. Angiology 2007;58:513-22.

2. Bots ML, Grobbee DE. Intima media thickness as a surrogate marker for generalised atherosclerosis. Cardiovasc Drugs Ther 2002;16:34151.

3. Gunes Y, Tuncer M, Guntekin U, Ceylan Y, Simsek H, Sahin M, Yildirim $M$. The relation between the color $M$-mode propagation velocity of the descending aorta and coronary and carotid atherosclerosis and flow-mediated dilatation. Echocardiography 2010;27:300-5.

4. Serruys PW, Morice MC, Kappetein AP, Colombo A, Holmes DR, Mack MJ, Stahle E, Feldman ET, Brand VM, Bass EJ, Dyck NV, Leadly K, Dawkins DK, Mohr FW. SYNTAX Investigators. Percutaneous coronary intervention versus coronary-artery bypass grafting for severe coronary artery disease. N Engl J Med 2009;360:961-72.

5. Valgimigli M, Serruys PW, Tsuchida K, Vaina S, Morel MA, van den Brand MJ, Colombo A, Morice MC, Dawkins K, Bruyne B, Boersma E. ARTS II. Cyphering the complexity of coronary artery disease using the syntax score to predict clinical outcome in patients with three-vessel lumen obstruction undergoing percutaneous coronary intervention. Am J Cardiol 2007;99:1072-81.

6. Chakrabarti AK, Gibson CM. The SYNTAX score: usefulness, limitations, and future directions. J Invasive Cardiol 2011;23:511-2.

7. Girasis C, Garg S, Raber L, Sarno G, Morel MA, Garcia-Garcia HM, Lüscher TF, Windecker S. SYNTAX score and clinical SYNTAX score as predictors of very long-term clinical outcomes in patients undergoing percutaneous coronary interventions: a substudy of SIRolimuseluting stent compared with pacliTAXel-eluting stent for coronary revascularization (SIRTAX) trial. Eur Heart J 2011;32:3115-27.

8. Korkmaz L, Bektas H, Korkmaz AA, Agaç MT, Acar Z, Erkan H, Celik S Increased carotid intima-media thickness is associated with higher SYNTAX score. Angiology 2012;63:386-89.

9. Stein JH, Korcarz CE, Hurst RT, Lonn E, Kendall CB, Mohler ER, Najjar SS. American Society of Echocardiography Carotid Intima-Media Thickness Task Force. Use of carotid ultrasound to identify subclinical vascular disease and evaluate cardiovascular disease risk: a consensus statement fromthe American Society of Echocardiography Carotid Intima-Media Thickness Task Force. Endorsed by the Society for Vascular Medicine. J Am Soc Echocardiogr 2008;21:93-111.

10. Sianos G, Morel MA, Kappetein AP, Morice MC, Colombo A, Dawkins $K$, Brand VM. The SYNTAX score: an angiographic tool grading the complexity of coronary artery disease. Eurointervention 2005;1:219 27.

11. Kim YH, Park DW, Kim WJ, Lee JY, Yun SC, Kang SJ, Lee WS, Lee WC, Park SJ. Validation of SYNTAX (Synergy between PCI with Taxus and cardiac surgery) score for prediction of outcomes after unprotected leftmain coronary revascularization. JACC Cardiovasc Interv 2010:3:612-23.

12. Chen SL, Ye F, Zhang JJ, Hu ZY, Tian NL, Zhang JX. Prediction of clinical outcomes in patients with unprotected left main trifurcation lesions treated by drug-eluting stents: Importance of 2-stent technique and SYNTAX score. J Interv Cardiol 2010;23:352-7.

13. Tanboga IH, Ekinci M, Isik T, Kurt M, Kaya A, Sevimli S. Reproducibility of syntax score: from core lab to real world. J Interv Cardiol 2011;24:302-6.

14. Aykan AÇ, Gül I, Gökdeniz T, Hatem E, Arslan AO, Kalaycıoğlu E, Turan T, Belen E, Cetin M. Ankle Brachial Index Intensifies the Diagnostic Accuracy of Epicardial Fat Thickness for the Prediction of Coronary Artery Disease Complexity. Heart Lung Circ 2014;23:764-71.

15. Aksakal E, Tanboga IH, Kurt M, Kaya A, Topcu S, Kalkan K, Sevimli Serdar. Predictors coronary lesions complexity in patients with stable coronary artery disease. Angiology 2013;64:304-9.

16. Duran M, Uysal OK, Gunebakmaz O, Baran O, Turfan M, Ornek E, Cetin M, Murat SN, Karadeniz M, Kurtul A, Kaya MG. Glomerular Filtration Rate is Associated With Burden of Coronary Atherosclerosis in Patients With Acute Coronary Syndrome. Angiology 2014;65:350-6.

17. Kirma C, Izgi A, Dundar C, Tanalp AC, Oduncu V, Aung SM, Sonmez K, Mutlu B, Özdemir N, Erentuğ V. Clinical and procedural predictors of no-reflow phenomenon after primary percutaneous coronary Interventions. Experience at a single center. Circ J 2008;72:716-21.

18. Baris N, Akdeniz B, Uyar S, Ozel E, Kirimli O, Badak O, Aslan O, Güneri $\mathrm{S}$. Are complex coronary lesions more frequent in patients with diabetes mellitus? Can J Cardiol 2006;22:935-7.

19. Thenappan T, Ali Raza J, Movahed A. Aortic atheromas: Current concepts and controversies-a review of the literature. Echocardiography 2008;25:198-207.

20. Simsek H, Sahin M, Gunes Y, Dogan A, Gumrukcuoglu HA, Tuncer M. A Novel Echocardiographic Method for the Detection of Subclinical Atherosclerosis in Newly Diagnosed, Untreated Type 2 Diabetes. Echocardiography 2013;30:644-8.

21. Fazio GP, Redberg RF, Winslow T, Schiller NB. Transesophageal echocardiographically detected atherosclerotic plaque is a marker for coronary artery disease. J Am Coll Cardiol 1993;21:144-150.

22. Kim HY, Kim CJ, Rho TH, Youn HJ, Jin SW, Rhim HY, Park SS Transesophageal echocardiographic detection of thoracic aortic plaque could noninvasively predict significant obstructive coronary artery disease. Korean J Intern Med 1999;14:20-6.

23. Gunes $Y$, Tuncer $M$, Yildirim M, Güntekin U, Gümrükçüoğlu HA, Sahin $M$. A novel echocardiographic method for the prediction of coronary artery disease. Med Sci Monit 2008;14:102-5.

24. Sen T, Tufekcioglu O, Ozdemir M, Tuncez A, Uygur B, Golbasi Z Kısacık H. A new echocardiographic parameter of aortic stiffness and atherosclerosis in patients with coronary artery disease: Aortic propagation velocity. J Cardiol 2013;62:236-40.

25. Ghaderi F, Samim H, Keihanian F, Danesh Sani SA. The predictive role of aortic propagation velocity for coronary artery disease. BMC Cardiovasc Disord 2018;18:121.

26. Burke GL, Evans GW, RileyWA, Sharrett AR, Howard G, BarnesRW, Rosamond W, Heiss G. Arterial wall thickness is associated with prevalent cardiovascular disease in middle-aged adults. The Atherosclerosis Risk in Communities (ARIC) Study. Stroke 1995:26:386-91. 